\title{
Intrinsic Religiosity Attenuates the Negative Relationship Between Social Disconnectedness and Meaning in Life
}

Forthcoming in Psychology of Religion and Spirituality

\author{
Caleb J. Reynolds a 1 \\ Spencer M. Smith ${ }^{\text {b, a } 2}$ \\ Paul Conway ${ }^{\text {a } 3}$
}

Note: This is the final submitted version of the manuscript, but it may differ slightly from the authoritative version of record published in Psychology of Religion and Spirituality. The authoritative version will be available on the publisher's website.

${ }^{a}$ Department of Psychology, Florida State University, 1107 W Call St., Tallahassee, FL 32306, USA

${ }^{\mathrm{b}}$ Department of Philosophy, Florida State University, 151 Dodd Hall, Tallahassee, FL 32306, USA

${ }^{1}$ creynolds@psy.fsu.edu (Corresponding Author)

2 sms13n@my.fsu.edu

${ }^{3}$ conway@psy.fsu.edu 


\begin{abstract}
Positive social connections are integral to people's experience of meaning in their lives. As such, social isolation can make life seem meaningless. Chan, Michalak, and Ybarra (2019, Journal of Personality) provided evidence that religious beliefs (not just participation in corporate religious life) can provide an alternative source of meaning for people who feel socially isolated. We tested whether this phenomenon was specific to (a) intrinsic vs. extrinsic orientations toward religion, and (b) experiencing meaning in one's life at present vs. actively searching for meaning. In a sample of undergraduates, high levels of intrinsic — but not extrinsic — religiosity attenuated the relationship between social disconnection and decreased meaning in life. Moreover, this attenuation was specific to felt presence of meaning in life, rather than active search for meaning. These results corroborate and expand Chan et al.'s findings and suggest that future research should explore mechanisms by which religious beliefs themselves serve meaning-making functions.
\end{abstract}

Keywords: meaning in life, social connection, religiosity, intrinsic religiosity 
Humans have a fundamental need to form and maintain strong interpersonal relationships (Baumeister \& Leary, 1995). It is perhaps unsurprising, then, that social connection contributes to the sense that one's life is meaningful (e.g., Baumeister \& Landau, 2018; Lambert et al., 2013). Conversely, social disconnection — insofar as it promotes loneliness - fosters a litany of negative physical and psychological consequences (e.g., Hawkley \& Cacioppo, 2010), including a decreased sense of meaning or purpose in life (Hicks \& King, 2009; Stillman et al., 2009).

In the face of social disconnection, religiosity may help buffer against such loss of meaning in life. There are at least two ways this might occur. First, religiosity might provide people with opportunities for meaningful social experiences - thereby directly addressing the isolation. Second, religion might not simply buffer the negative effects of isolation via increased social exposure, but rather religious beliefs themselves may provide an alternate source of meaning both directly, by providing a greater sense of purpose in the world, and indirectly, by providing an alternative source of connection: felt communion with the divine. Chan and colleagues (2019) investigated this latter possibility in nationally-representative samples and found that religious beliefs attenuated the link between social disconnection and decreased purpose in life over and above religious attendance.

These meaning-making features of religion mirror a distinction between intrinsic and extrinsic religious motivations. People high on intrinsic religiosity focus on religion for its own sake, integrating religion into all of their lives; people high on extrinsic religiosity focus on religion's instrumental uses, such as social connections or personal comfort (Cohen et al., 2017). Contrasting these two orientations toward religion enables a test of whether religious beliefs in particular, rather than religious social interactions, buffer the negative effect of social disconnection on meaning in life. For if participation in the life of a religious community buffers 
the effects of social disconnection via increased opportunities for social interactions, then the attenuation effect observed by Chan et al. (2019) should primarily reflect extrinsic religiosity, which taps social motivations for engaging in religion. Conversely, if Chan et al. are correct that religious beliefs themselves have a meaning-making role (beyond simply promoting social interactions), then the attenuation effect should primarily reflect intrinsic religiosity.

Additionally, meaning in life can be conceptualized both in terms of how much a person feels that their life has meaning now-presence of meaning — and in terms of how much a person is actively seeking meaning in the world-search for meaning. Although Chan et al. measured present purpose in life, it is possible that social disconnection also motivates people to actively search for an alternative source of meaning.

In the current work, we utilized unpublished data from our file drawer to test whether the attenuation pattern observed by Chan et al. (2019) replicates, and whether it is specific to (a) intrinsic vs. extrinsic religiosity, and (b) presence of meaning in life vs. search for meaning in life. We anticipated replicating the finding that religiosity attenuates the negative relationship between social disconnection and meaning in life (Chan et al., 2019). However, theorizing by Chan et al. suggests this pattern should be specific to intrinsic (rather than extrinsic) religiosity and should emerge primarily for presence of meaning, rather than search for meaning.

\section{Method}

Between the manuscript and supplement, we report how we determined sample size, all exclusions, and all measures. Study protocols were approved by the Florida State University institutional review board. Data, syntax, and materials are available at osf.io/2yhr8.

\section{Participants}

We collected the present data for hypotheses unrelated to this paper, collecting as many 
participants as possible in two academic semesters. Eight hundred twenty-eight undergraduates from a large public university in the southeastern U.S. participated online for course credit, and we analyzed responses from 797 participants who provided complete data (177 men, 620 women). A sensitivity analysis indicated that this sample size provided $88 \%$ power to detect an interaction effect of $f^{2}=.02$ in a model with 5 predictors. Most participants were Protestant (39.5\%), Catholic (30.2\%), atheist/agnostic (7.8\%), Jewish (5.6\%), or religiously unaffiliated (12.3\%), and were Caucasian (75.5\%), Hispanic/Latino (20.3\%), or African American (10.0\%).

\section{Measures}

Participants completed the 14-item Revised Intrinsic/Extrinsic Religiosity Scale (Gorsuch \& McPherson, 1989) ${ }^{1}$, the 8-item Social Connectedness Scale (Lee \& Robbins, 1995), and the two 5-item subscales (presence of meaning and search for meaning) of the Meaning in Life Questionnaire (Steger et al., 2006) on 7-point scales. We inverted the Social Connectedness Scale so higher scores represent greater social disconnection.

\section{Results \& Discussion}

We present descriptive statistics and correlations in Table 1. To test our hypotheses, we analyzed two linear models predicting a) presence of meaning in life, and b) search for meaning in life. As predictors in each model, we included social disconnectedness, intrinsic religiosity, extrinsic religiosity, and the two-way interactions between social disconnectedness and intrinsic and extrinsic religiosity, standardizing both predictors and outcomes for analysis. ${ }^{2}$ If religious

\footnotetext{
${ }^{1}$ This measure might be problematic for use among religiously unaffiliated participants. See the Supplement for a discussion of this point, as well as robustness checks indicating key results are unchanged when using an alternative scoring of this measure and when excluding religiously unaffiliated participants. We would like to thank an anonymous reviewer for pressing us to clarify this issue.

${ }^{2}$ We standardized variables due to multicollinearity issues (variance inflation factors from 4.9-18.0 raw, 1.0-1.5 standardized). Standardizing when modeling interactions can reduce variance inflation factors and change results for lower order terms, but does not affect inferences based on the interaction terms (Hayes, 2013). Thus, this choice does not affect the key results: interactions between intrinsic/extrinsic religiosity and social disconnection.
} 
beliefs themselves buffer the relationship between social disconnection and meaning in life, intrinsic, but not extrinsic, religiosity should interact with social disconnection.

First, we examined the model for presence of meaning in life (Table 2, Figure 1A\&B). Consistent with Chan et al. (2019), intrinsic religiosity attenuated the negative effect of social disconnection on presence of meaning in life, $t(791)=3.26, p=.001$, (simple slopes: $\beta=-.36$ and -.60 at +/-1SD of intrinsic religiosity, respectively). A Johnson-Neyman analysis clarified that the negative relationship between social disconnectedness and meaning in life was significant when intrinsic religiosity was less than $Z=2.43$ (in our data, less than $1 \%$ of the sample; corresponds to $\sim 6.77$ on the 7 point scale), suggesting that while intrinsic religiosity may attenuate the relationship, it likely does not eliminate it. In contrast, extrinsic religiosity did not attenuate the negative relationship between social disconnection and presence of meaning in life, $t(791)=-1.11, p=.268$ (simple slopes: $\beta=-.52$ and -.44 at $+/-1$ SD of extrinsic religiosity). These model results suggest that, at least for presence of meaning in life, the buffering effect of religion on presence of meaning in life seems to reflect motivations to engage in religion for its own sake, rather than for social purposes.

Second, we examined the search for meaning in life model (Table 2, Figure 1C\&D). Neither intrinsic nor extrinsic religiosity interacted with social disconnectedness to predict search for meaning in life, $t(791)=0.18, p=.861$ (simple slopes: $\beta=.19$ and .18 at $+/-1$ SD of intrinsic religiosity), and $t(791)=-0.86, p=.392$ (simple slopes: $\beta=.15$ and .22 at $+/-1$ SD of extrinsic religiosity), respectively. This suggests religion's effect on the relationship between social disconnection and meaning in life is not about motivating an ongoing search for meaning. Patterns held when including demographic covariates, excluding religiously unaffiliated participants, and using alternative scoring for intrinsic/extrinsic religiosity (see Supplement). 
Together, our results corroborate and extend the findings from Chan et al. (2019). As in their findings, religiosity attenuated the negative relationship between feelings of social isolation and perceptions of meaning in one's life. However, our results clarify that this attenuation may be specific to an orientation of "religion for religion's sake," — intrinsic religiosity — rather than an orientation of religion for the sake of social ends - extrinsic religiosity. As noted in the Introduction, if religion gives meaning primarily by providing social opportunities, our results should reflect extrinsic religiosity. Alternatively, if religious beliefs themselves give meaning our results should reflect intrinsic religiosity, as indeed they do. Therefore, our results are consistent with Chan et al.'s hypothesis that religious beliefs — rather than religious social opportunitiescan buffer the loss of meaning in life that comes with social isolation.

Moreover, this pattern of effects emerged for presence of, but not search for, meaning in life, suggesting that intrinsic religiosity is not spurring a greater search for meaning among socially disconnected people, but rather is making meaning in the here and now. As we used existing data, we could not compare the two potential pathways Chan et al. (2019) propose: a greater sense of meaning and purpose in the world and an increased sense of communion with the divine. Future work might profitably examine mechanisms of this effect.

The present findings are limited by our use of a convenience sample of undergraduates, who may have a narrow set of meaning-making life experiences (compared to representative samples) and who report high social connection. Additionally, we did not measure religious attendance, limiting our ability to rule out the plausible alternative that religiosity attenuates links between social disconnection and meaning via increased social opportunities. Although extrinsic religiosity taps motivations to engage in religious activity for social and personal instrumental reasons, it likely does not tap the actual behaviors of interacting with others in religious settings. 
Thus, extrinsic religiosity might not capture the extent to which participation in a religious community is integral to religiosity's positive effects on meaning in life. Finally, intrinsic religiosity in this study may be serving as a proxy for some other variable that buffers the relationship between disconnection and meaning in life, such as positive affect (Hicks et al., 2010), and we cannot rule out such possible third variables in the current data.

Intrinsic religiosity attenuated the negative relationship between social disconnection and felt presence of meaning in life, but extrinsic religiosity did not. It seems that for the socially isolated, religiosity's meaning-making power lies in directing one's life, rather than motivating social connection. 
Table 1

Descriptive Statistics and Correlations for Primary Variables of Interest

\begin{tabular}{|c|c|c|c|c|c|c|c|c|c|}
\hline Variable & $M$ & $S D$ & $\alpha$ & 1 & 2 & 3 & 4 & 5 & 6 \\
\hline $\begin{array}{l}\text { 1. Intrinsic } \\
\text { Religiosity }\end{array}$ & 3.73 & 1.25 & .77 & & & & & & \\
\hline $\begin{array}{l}\text { 2. Extrinsic } \\
\text { Religiosity }\end{array}$ & 2.92 & 1.32 & .84 & $.55^{\star \star \star}$ & & & & & \\
\hline $\begin{array}{l}\text { 3. Lack of Social } \\
\text { Connectedness }\end{array}$ & 2.02 & 1.22 & .94 & -.05 & .03 & & & & \\
\hline $\begin{array}{l}\text { 4. Presence of } \\
\text { Meaning in Life }\end{array}$ & 5.02 & 1.23 & .86 & $.25^{\star \star \star}$ & $.16^{\star \star *}$ & $-.49^{\star * *}$ & & & \\
\hline $\begin{array}{l}\text { 5. Search for } \\
\text { Meaning in Life }\end{array}$ & 4.95 & 1.39 & .89 & $-.10^{\star \star}$ & -.01 & $.19^{\star \star \star}$ & $-.15^{\star \star \star}$ & & \\
\hline 6. Age & 19.27 & 1.60 & -- & -.02 & -.04 & .03 & -.03 & .02 & \\
\hline $\begin{array}{l}\text { 7. Gender } \\
\text { (1=women, } 2=\text { men })\end{array}$ & 1.22 & 0.42 & -- & -.05 & $-.10^{\star \star}$ & .04 & -.05 & $-.07^{\star}$ & .02 \\
\hline
\end{tabular}

Note. $M, S D$, and $\alpha$ represent mean, standard deviation, and Cronbach's alpha, respectively. Statistically significant results are bolded for emphasis. $* p<.05, * * p<.01, * * * p<.001$. 
Table 2

Regression Results for Models Predicting Presence of and Search for Meaning in Life

\begin{tabular}{|c|c|c|c|c|c|c|c|c|}
\hline \multirow[t]{2}{*}{ Outcome: } & \multicolumn{4}{|c|}{ Presence of Meaning in Life } & \multicolumn{4}{|c|}{ Search for Meaning in Life } \\
\hline & $\beta$ & $\begin{array}{l}\beta 95 \% \mathrm{Cl} \\
{[\mathrm{LL}, \mathrm{UL}]}\end{array}$ & $s r^{2}$ & $\begin{array}{c}s r^{2} \\
95 \% \mathrm{Cl} \\
{[\mathrm{LL}, \mathrm{UL}]}\end{array}$ & $\beta$ & $\begin{array}{l}\beta \text { 95\% Cl } \\
{[\mathrm{LL}, \mathrm{UL}]}\end{array}$ & $s r^{2}$ & $\begin{array}{c}s r^{2} \\
95 \% \mathrm{Cl} \\
{[\mathrm{LL}, \mathrm{UL}]}\end{array}$ \\
\hline (Intercept) & 0.01 & {$[-0.05,0.07]$} & & & 0.00 & {$[-0.07,0.07]$} & & \\
\hline Social Disconnectedness & $-0.48^{\star \star \star}$ & {$[-0.54,-0.42]$} & .23 & {$[.18, .28]$} & $0.19^{\star \star \star}$ & {$[0.12,0.25]$} & .03 & {$[.01, .06]$} \\
\hline Intrinsic Religiosity & $0.21^{* \star *}$ & {$[0.14,0.29]$} & .03 & {$[.01, .05]$} & $-0.12^{\star *}$ & {$[-0.21,-0.04]$} & .01 & {$[-.00, .02]$} \\
\hline Extrinsic Religiosity & 0.06 & {$[-0.01,0.13]$} & .00 & {$[-.00, .01]$} & 0.06 & {$[-0.02,0.14]$} & .00 & {$[-.00, .01]$} \\
\hline $\begin{array}{l}\text { Social Disconnectedness } \\
\times \text { Intrinsic Religiosity }\end{array}$ & $0.12^{\star \star}$ & {$[0.05,0.19]$} & .01 & {$[-.00, .02]$} & 0.01 & {$[-0.08,0.09]$} & .00 & {$[-.00, .00]$} \\
\hline \multirow[t]{3}{*}{$\begin{array}{l}\text { Social Disconnectedness } \\
\times \text { Extrinsic Religiosity }\end{array}$} & -0.04 & {$[-0.10,0.03]$} & .00 & {$[-.00, .00]$} & -0.03 & {$[-0.11,0.04]$} & .00 & {$[-.00, .00]$} \\
\hline & & & & $R^{2}=.306^{\star \star \star}$ & & & & $R^{2}=.049^{\star \star \star}$ \\
\hline & & & & $95 \% \mathrm{CI}[.25, .35]$ & & & & $95 \% \mathrm{Cl}[.02, .08]$ \\
\hline
\end{tabular}

Note. $\beta$ : standardized regression weights. $s r^{2}$ : semi-partial correlation squared. $L L$ and $U L$ : lower and upper limits of a confidence interval. Statistically significant results are bolded for emphasis.

$* * p<.01, * * * p<.001$. 

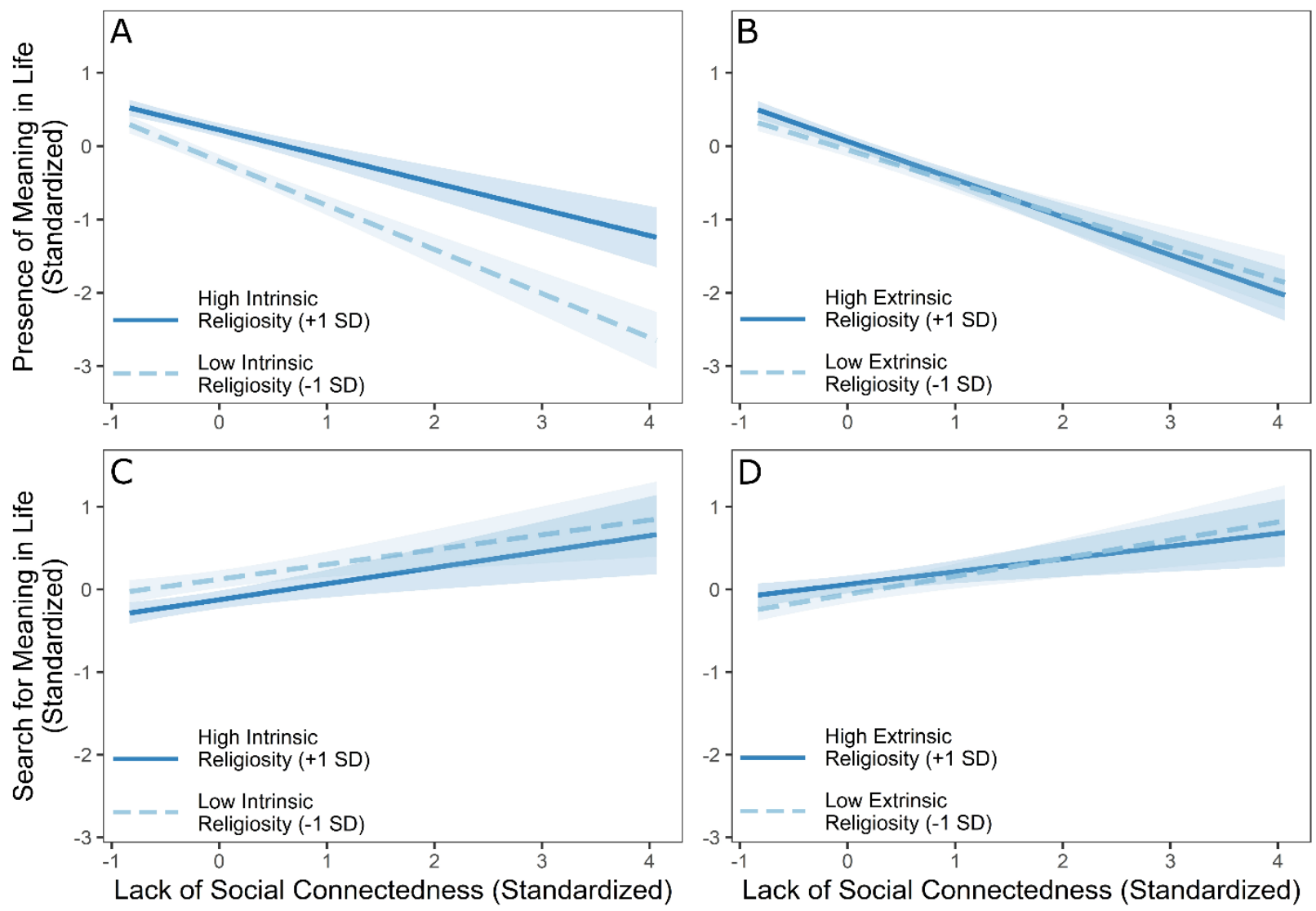

Figure 1. Interaction effects of social connectedness with intrinsic/extrinsic religiosity to predict presence of $(\mathrm{A} \& \mathrm{~B})$ and search for $(\mathrm{C} \& \mathrm{D})$ meaning in life. Bands around regression lines denote $95 \%$ confidence intervals. Axes are scaled to the minimum and maximum observed values. 


\section{Supplement to Intrinsic Religiosity Attenuates the Negative Relationship Between Social Disconnectedness and Meaning in Life}

\section{Supplementary Method}

In addition to the measures reported in the main text, participants also completed a pair of measures thought to tap lay moral realism and a single-item measure of religiosity. All materials are available at osf.io/2yhr8.

Participants completed Goodwin and Darley’s (2012) moral objectivism procedure. Based on previous theorizing, Goodwin and Darley $(2008,2010,2012)$ took lay moral objectivism to involve two beliefs: a) moral claims have truth values, and b) when two moral claims compete, one must be wrong. To generate scores for moral objectivism, participants read scenarios featuring a variety of moral acts and provide responses to questions targeting the two beliefs thought to be necessary for objectivism. In the present study, participants responded to the six moral transgressions from Goodwin and Darley (2012), which involved stealing, assaulting, lying, desecrating, performing a disrespectful gesture, and burning a flag. For each scenario, participants rated three features on scales with no midpoint. Participants indicated their agreement the claim that the act was morally wrong (1: Strongly disagree, 6: Strongly agree) and rated the extent to which they thought there was a single correct answer about whether or not the act was wrong (i.e., the claim has a truth value; 1: No correct answer, 6: Definitely a correct answer). Participants were then told to suppose that another participant gave the opposite of their evaluation of the wrongness of the act and rated the extent to which they thought the other person must be mistaken, as opposed to neither party being mistaken (1: Neither of us need be mistaken, 6: Other person is clearly mistaken). Following Goodwin and Darley (2012), we 
averaged these latter two assessments for all claims to generate a general objectivism score for each participant, with higher scores corresponding to more objectivist views.

Participants also completed the moral relativism subscale from Forsyth's (1980) Ethics Position Questionnaire. Because the original hypotheses for this dataset involved moral absolutism, thought to be the inverse of relativism, we scored the Ethics Position Questionnaire such that higher scores denote less relativistic responses (i.e., more absolutist responses).

Finally, participants rated their degree of religiosity on a single 7-point Likert scale ranging from not religious at all (1), to extremely religious (7).

We used G*Power (Faul, Erdfelder, Lang, \& Buchner, 2007) for the sensitivity analysis and $\mathrm{R}$ for all other analyses (Version 3.5.3; R Core Team, 2017), specifically the R-packages apaTables (Version 2.0.5; Stanley, 2018), car (Version 3.0-3; Fox \& Weisberg, 2019), haven (Version 2.0.0; Wickham \& Miller, 2018), jtools (Version 1.1.1; Long, 2018), and tidyverse (Version 1.2.1; Wickham, 2017). Syntax is available at osf.io/2yhr8.

\section{Supplementary Results}

We present descriptive statistics and full correlations among all measures in Table S1. To check the robustness of the pattern of results reported in the main text, we analyzed a series of alternative models. First, we examined whether results were robust to the inclusion of demographic covariates, and whether results emerged when using a single-item measure of global religiosity. Moreover, given issues in the measurement of intrinsic religiosity among participants who were religiously unaffiliated, we analyzed several models to ensure that such participants were not artificially driving our results. These models excluded nonreligious participants according to multiple criteria, and utilized alternative scoring of our key moderators, intrinsic and extrinsic religiosity. 


\section{Demographic Covariates and Single-Item Religiosity}

First, we reanalyzed the models in the main text while controlling for demographic predictors that were adequately represented in our sample: age, gender, religious affiliation (Protestant, Catholic, Jewish, No religious affiliation), and race/ethnicity (White, Black, Hispanic). We effect-coded religious affiliation and race/ethnicity, so regression coefficients for those variables represent deviations from the grand mean (Darlington \& Hayes, 2017).

Controlling for these covariates did not change the pattern of results: Intrinsic religiosity still significantly attenuated the relationship between social disconnection and presence of meaning in life, but this effect did not emerge for extrinsic religiosity or for search for meaning in life (Table S2).

Second, we analyzed a pair of models using the single-item measure of religiosity, rather than intrinsic and extrinsic religiosity. Specifically, we tested models predicting presence of meaning in life and search for meaning in life from religiosity, social disconnection, and their interaction (Table S3). In these single-item models, religiosity did not significantly attenuate the negative relationship between social disconnection and either presence of or search for meaning in life. It is unclear what this difference in results implies. It is highly plausible that this difference simply reflects the fact that this single-item measure is less sensitive than our multiitem measure of intrinsic religiosity. However, it might also reflect a meaningful difference between global evaluations of religiosity and more specific evaluations of religiosity, such as specific motivational orientations toward religion (as in the main text) or religious beliefs separate from religious attendance (as in Chan, Michalak, \& Ybarra, 2019). Future work might profitably clarify this point. 


\section{Measuring Intrinsic Religiosity Among Religious "Nones"}

Prior researchers have noted that the particular wording of some of the intrinsic religiosity items made it difficult for people without a religious affiliation (i.e., religious "nones"; Pew Research Center, 2015) to know whether to indicate that they agree or disagree with the items, thus making it difficult to complete the measure (e.g., Maltby, McCollam, \& Millar, 1994). Accordingly, Maltby and Lewis (1996) tested whether issues with noncompletion on the Age-Universal Intrinsic/Extrinsic Religiosity Scale (Gorsuch \& Venable, 1983) could be ameliorated by leading the items with the instruction "Think about each item carefully, Does the attitude or behavior described in the statement apply to me?” (p. 940) and using a three-point scale formed of No (1), Not certain (2), and Yes (3). Indeed, they found that using these applicability response scales (rather than agreement scales) virtually eliminated noncompletion of the measure and improved internal consistency. They took this as evidence that amending the response scale for this measure made it appropriate for use among nonreligious participants. ${ }^{3}$

Following Maltby and Lewis' recommendations, in the present study, we had participants complete the Revised Intrinsic/Extrinsic Religiosity Scale (Gorsuch \& McPherson, 1989; revised from the measure by Gorsuch \& Venable, 1983) on seven-point scales anchored at Does not apply to me at all (1) and Strongly applies to me (7). Maltby and Lewis (1996) suggested threepoint scales as described above, but we used seven-point scales to increase sensitivity and reliability (e.g., Maitland, 2009). The intrinsic religiosity subscale of this measure contains five normally worded items and three negatively worded items. Internal consistency of the eight intrinsic religiosity items was adequate, $\alpha=.77$ (Table 1 in the main text).

\footnotetext{
${ }^{3}$ Maltby and Day (1998) later made the same argument for revising Batson's measure of Quest religious orientation (Batson \& Schoenrade, 1991).
} 
However, on the suggestion of an anonymous reviewer, we examined internal consistency of the intrinsic religiosity scale separately among participants who were religiously unaffiliated (i.e., those who identified their current religious affiliation as Atheist/agnostic or Nothing in particular) and religious affiliated (all others). Among religiously affiliated participants, intrinsic religiosity exhibited good internal consistency $(\alpha=.83)$, but among religiously unaffiliated participants, internal consistency approximated random responding $(\alpha=$ .02 , not a typo). Principal axis factor analyses (with oblimin rotation) splitting the sample by religious affiliation (affiliated vs. unaffiliated) revealed that the three reverse-coded items performed differently in the two groups. In both groups, the eight intrinsic religiosity items loaded onto two factors, one each for the positively and negatively worded items ( 5 and 3 items, respectively). However, in the religiously affiliated group the two factors positively correlated, $r$ $=.25$, whereas in the religiously unaffiliated group the two factors negatively correlated, $r=-.62$. Consequently, a composite of the positively worded items was positively correlated with the a composite of the (recoded) negatively worded items for religiously affiliated participants, $r(635)$ $=.26, p<.001$, but the two composites were strongly negatively correlated for religiously unaffiliated participants, $r(158)=-.50, p<.001^{4}$

Thus, although participants with no religious affiliation could complete the measure with the applicability anchors, they apparently did so in a manner that reversed the meaning of the

\footnotetext{
${ }^{4}$ We replicated this pattern of results in a separate sample of 469 MTurkers who completed the same items using the same instructions and response scales as in the present study (Reynolds, Gamez-Djokic, Molden, \& Conway, in prep, Study 3$)$. The five positively worded items hung together well among both religiously affiliated $(\alpha=.89)$ and unaffiliated $(\alpha=.86)$ participants, but internal consistency of the full 8-item measure was good among religiously affiliated participants $(\alpha=.84)$ and abysmal among religiously unaffiliated participants $(\alpha=.20)$. Moreover, this reflected the fact that the positively and negatively worded sets of items correlated positively among religiously affiliated participants $r(250)=.42, p<.001$, but negatively among religiously unaffiliated participants, $r(215)=-$ $.40, p<.001$. Thus, the issues with this intrinsic religiosity measure among nonreligious participants are not specific to the present sample or to undergraduate samples more generally.
} 
items relative to the same items among religiously affiliated participants. Examination of the three negatively worded items suggests some direction as to why these items are problematic:

1. Although I believe in my religion, many other things are more important in life.

2. Although I am religious, I don't let it affect my daily life.

3. It doesn't much matter what I believe so long as I am good.

The issue with the first and second items is almost certainly that they are double-barreled: They explicitly include statements that one is religious in addition to statements that get at the intrinsic motivation component. Thus, when reverse-scoring these items, a person who is not religious can easily say statements 1 and 2 do not apply to them (because they are not, in fact, religious, as the statement says), and thus end up with a high intrinsic religiosity score on these items once they are recoded. Indeed, $66.3 \%$ and $78.1 \%$ of religiously unaffiliated participants selected the lowest value "1 - Does not apply to me at all" for these two items (respectively). The issue with the third item is a little less obvious, and indeed, only $22.5 \%$ of religiously unaffiliated participants endorsed the lowest value (and thus received the highest score on this item). The rest of the responses to item three were heavily negatively skewed for religiously unaffiliated participants, as one would expect if this item functions as a reverse-scored intrinsic religiosity item. The issue with this item might be that some participants who encounter this item in the middle of a religiosity scale interpret "what I believe" to necessarily include religious beliefs, which they don't have (hence, "Does not apply to me"), and for such participants the item effectively becomes double-barreled in the same way as the first two items. Thus, when using Maltby and Lewis' (1996) suggestion for anchors, the negatively worded items do not function appropriately among nonreligious participants, and we recommend that other researchers do not 
employ these items, especially with the applicability wording suggested by Maltby and Lewis (1996).

We are certainly not the first to note this issue with intrinsic religiosity scales. Notably, Cohen and colleagues (2017) examined measurement properties of the Revised Intrinsic/Extrinsic Religiosity Scales and noted that the negatively worded items worsened model fit for intrinsic religiosity and were double-barreled. Thus, they suggested excluding such items from scoring the Revised Intrinsic/Extrinsic Religiosity Scales. However, the extent of the issue we observed here may not have been visible in their data, which consisted of religious samples.

\section{Excluding Religiously Unaffiliated Participants}

To ascertain the effects of these measurement issues on our present results, we analyzed several alternative models. The key pattern of results - the negative relationship between social disconnectedness and presence of meaning in life is attenuated among those high in intrinsic religiosity — held when excluding just atheists and agnostics (Table S4), and when excluding anyone who was religiously unaffiliated (Table S5), despite the fact that these exclusions restrict the variance in intrinsic and extrinsic religiosity, and may restrict the variance in meaning in life. ${ }^{5}$ Moreover, as in the primary analyses reported in the main text, this same attenuation was not observed for extrinsic religiosity or for search for meaning in life (Tables S4 \& S5).

\section{Alternative Scoring of Religiosity Measures}

More directly, we reanalyzed the data following Cohen and colleagues' (2017) recommendations for scoring the Revised Intrinsic/Extrinsic Religiosity Scale. Cohen et al. argue

\footnotetext{
${ }^{5}$ As noted by an anonymous reviewer, prior work suggests that meaning in life is higher among the religiously affiliated than the unaffiliated. Indeed, participants who were religiously affiliated $(n=637)$ were higher on presence of meaning in life $(M=5.16, S D=1.18)$ than participants who were religiously unaffiliated $(n=160, M=$ $4.46, S D=1.25), t(795)=6.71, p<.001$, and lower on search for meaning in life $(M=4.87, S D=1.42)$ vs $(M=$ $5.27, S D=1.19), t(795)=-3.27, p=.001$. Thus, excluding religiously unaffiliated participants from analyses restricted the variance of intrinsic religiosity ( $\sigma^{2}$ from 3.24 to 2.78 ) and slightly restricted the variance of presence of meaning in life ( $\sigma^{2}$ from 1.51 to 1.40$)$, yet the primary effects held regardless.
} 
that the measure is better scored as a 3 -factor scale in which negatively worded intrinsic religiosity items are dropped and extrinsic religiosity is split into separate components for social and personal motivations. This scoring format offers two key advances for the present project.

First, scoring just the five positively worded items should make the intrinsic religiosity scale more sensitive among religiously unaffiliated participants, as relationships between intrinsic religiosity and meaning in life will not be attenuated by group-specific unreliability. Second, splitting extrinsic religiosity into social and personal motivations might give a cleaner test of the competing hypothesis that the relationship between social disconnection and meaning in life is attenuated not by religious beliefs per se, but rather by people using religion as a means to fulfill social needs.

Therefore, we analyzed a pair of models in which these three factors (intrinsic religiosity [less the three negatively worded items], social extrinsic religiosity, and personal extrinsic religiosity) each interacted with social disconnection to predict presence of meaning in life and search for meaning in life (Table S6). As in the primary results reported in the main text, intrinsic religiosity attenuated the relationship between social disconnection and presence of meaning in life, but this effect was not observed for search for meaning in life, and neither social nor personal extrinsic religiosity interacted with social disconnection to predict either presence of or search for meaning in life (Table S6). Moreover, this same pattern largely held when excluding atheists and agnostics (Table S7), as well as when excluding atheists, agnostics, and people with no particular religious affiliation (Table S8): Intrinsic religiosity attenuated the relationship between social disconnectedness and presence of meaning in life in both models, but the model excluding atheists and agnostics suggested that the same relationship might be slightly enhanced among people high in personal extrinsic religiosity (Table S7). 
The results from these models suggest two conclusions: 1) the key pattern of results is not driven by religiously unaffiliated participants who score low on intrinsic religiosity, and 2) the measurement issue among such participants is not dramatically influencing the key results. Thus, we feel confident that our results are not driven by either measurement issues among nonreligious participants or by the lower meaning in life among nonreligious participants. Because the results do not change when analyzing in these alternative manners, we retain the models employing the original scoring of intrinsic and extrinsic religiosity in the main text.

\section{Conclusion}

Together, the several alternative models reported in this Supplement lend confidence to our interpretation of our primary findings. Intrinsic religiosity's attenuation of the negative relationship between social disconnection and presence of meaning in life held when controlling for a variety of demographic covariates, when excluding nonreligious participants (for whom the measure of intrinsic religiosity might be problematic), and when using alternative scoring methods for intrinsic and extrinsic religiosity, though the effect did not emerge when using a single item measure of religiosity. 
Table S1

Descriptive Statistics and Correlations for All Variables

\begin{tabular}{|c|c|c|c|c|c|c|c|c|c|c|c|c|}
\hline Variable & $M$ & $S D$ & $\alpha$ & 1 & 2 & 3 & 4 & 5 & 6 & 7 & 8 & 9 \\
\hline $\begin{array}{l}\text { 1. Religiosity } \\
\text { (Single Item) }\end{array}$ & 3.50 & 1.83 & -- & & & & & & & & & \\
\hline $\begin{array}{l}\text { 2. Intrinsic } \\
\text { Religiosity }\end{array}$ & 3.73 & 1.25 & .77 & $.76^{\star \star \star}$ & & & & & & & & \\
\hline $\begin{array}{l}\text { 3. Extrinsic } \\
\text { Religiosity }\end{array}$ & 2.92 & 1.32 & .84 & $.70^{\star \star \star}$ & $.55^{\star \star \star}$ & & & & & & & \\
\hline $\begin{array}{l}\text { 4. Lack of Social } \\
\text { Connectedness }\end{array}$ & 2.02 & 1.22 & .94 & $-.09^{\star}$ & -.05 & .03 & & & & & & \\
\hline $\begin{array}{l}\text { 5. Presence of } \\
\text { Meaning in Life }\end{array}$ & 5.02 & 1.23 & .86 & $.26^{\star \star \star}$ & $.25^{\star \star \star}$ & $.16^{\star \star \star}$ & $-.49^{\star \star \star}$ & & & & & \\
\hline $\begin{array}{l}\text { 6. Search for } \\
\text { Meaning in Life }\end{array}$ & 4.95 & 1.39 & .89 & $-.08^{\star}$ & $-.10^{\star \star}$ & -.01 & $.19^{\star \star \star}$ & $-.15^{\star \star \star}$ & & & & \\
\hline $\begin{array}{l}\text { 7. Moral } \\
\text { Absolutism }\end{array}$ & 3.99 & 0.96 & .84 & $.20^{\star \star \star}$ & $.23^{\star \star \star}$ & .06 & $-.14^{\star \star \star}$ & $.14^{\star \star \star}$ & $-.14^{\star \star \star}$ & & & \\
\hline $\begin{array}{l}\text { 8. Moral } \\
\text { Objectivism }\end{array}$ & 5.32 & 1.21 & .88 & $.11^{\star \star}$ & $.10^{\star \star}$ & .05 & -.06 & $.12^{\star \star \star}$ & .07 & $.22^{\star \star \star}$ & & \\
\hline 9. Age & 19.27 & 1.60 & -- & -.02 & -.02 & -.04 & .03 & -.03 & .02 & -.04 & .00 & \\
\hline $\begin{array}{l}\text { 10. Gender } \\
(1=w, 2=m)\end{array}$ & 1.22 & 0.42 & -- & $-.08^{\star}$ & -.05 & $-.10^{\star \star}$ & .04 & -.05 & $-.07^{*}$ & $-.08^{\star}$ & -.01 & .02 \\
\hline
\end{tabular}

Note. $M, S D$, and $\alpha$ represent mean, standard deviation, and Cronbach's alpha, respectively. Values in square brackets indicate the $95 \%$ confidence interval for each correlation. Statistically significant results are bolded for emphasis.

$*$ indicates $p<.05, * *$ indicates $p<.01, * * *$ indicates $p<.001$. 
Table S2

Regression Results Predicting Presence of and Search for Meaning with Demographic Covariates Included

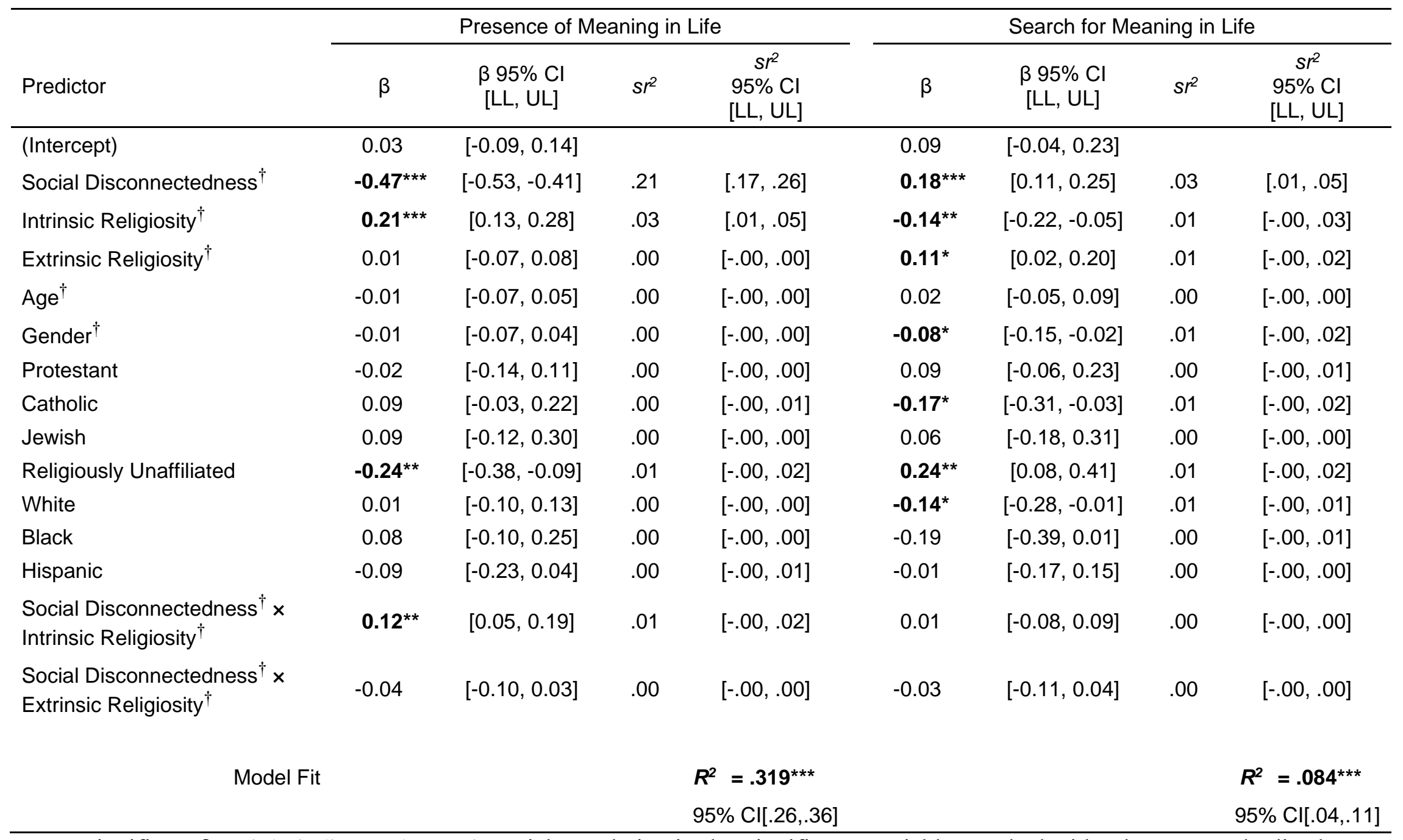

Note. A significant $\beta$-weight indicates the semi-partial correlation is also significant. Variables marked with a $\dagger$ were standardized prior to analysis, as were the outcome variables. Religious affiliation (Protestant, Catholic, Jewish, and unaffiliated) and race/ethnicity 
(White, Black, Hispanic) are effect-coded, so these predictors represent deviations from the grand mean. $s r^{2}$ represents the semi-partial correlation squared. $L L$ and $U L$ indicate the lower and upper limits of a confidence interval, respectively. Statistically significant results are bolded for emphasis.

$*$ indicates $p<.05, * *$ indicates $p<.01, * * *$ indicates $p<.001$. 
Table S3

Regression Results Predicting Presence of and Search for Meaning Based on a Single-Item Measure of Religiosity

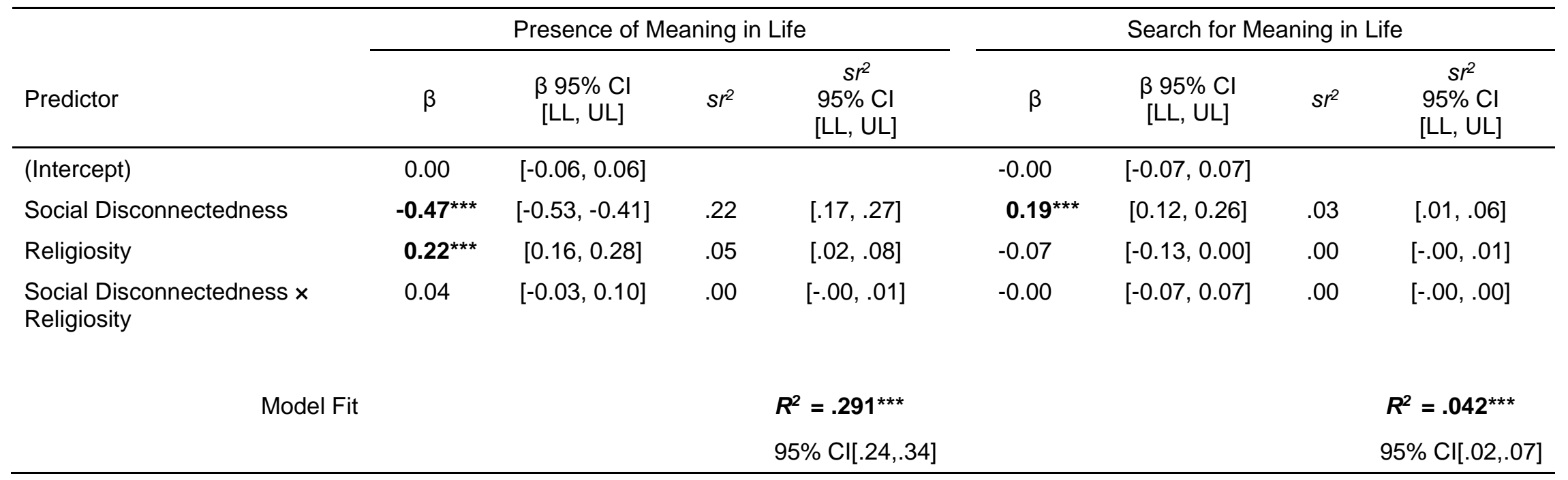

Note. A significant $\beta$-weight indicates the semi-partial correlation is also significant. All variables in the model are standardized, so $\beta$ represents standardized regression weights. $s r^{2}$ represents the semi-partial correlation squared. $L L$ and $U L$ indicate the lower and upper limits of a confidence interval, respectively. Statistically significant results are bolded for emphasis.

$* * *$ indicates $p<.001$. 
Table S4

Regression Results Predicting Presence of and Search for Meaning, Excluding Atheists and Agnostics $(\mathrm{N}=735)$

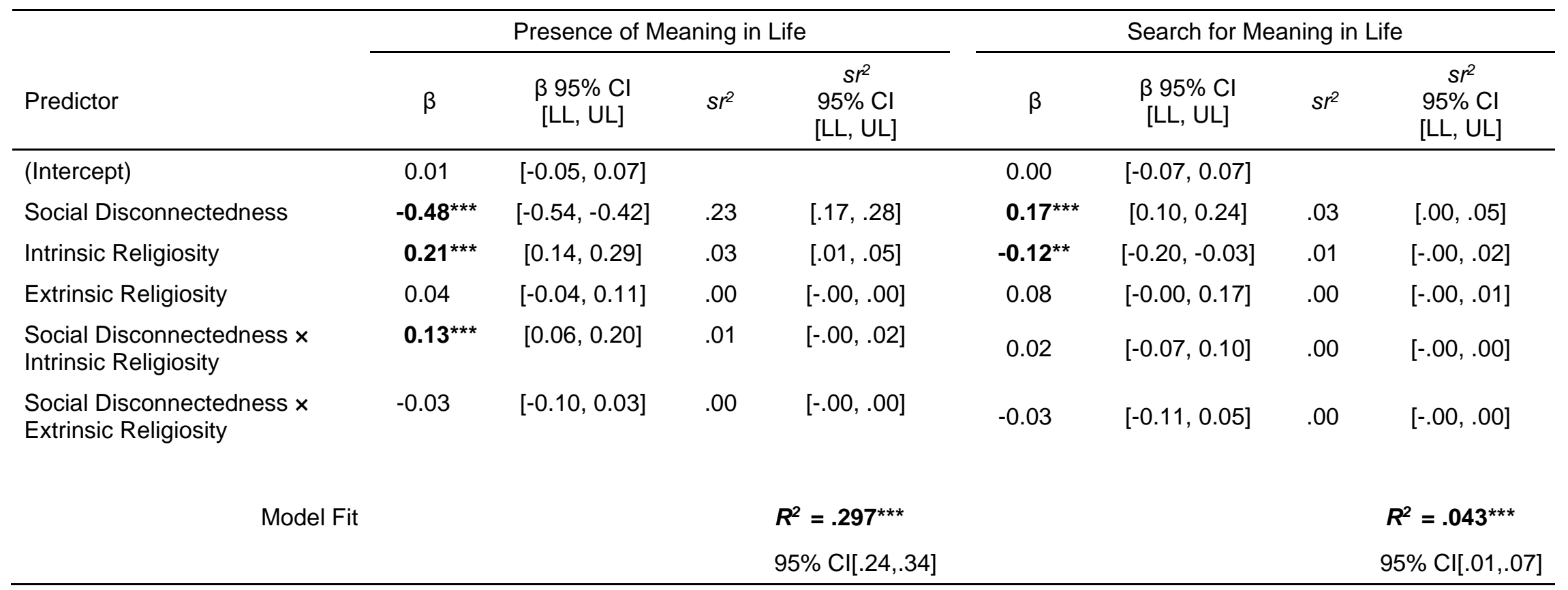

Note. A significant $\beta$-weight indicates the semi-partial correlation is also significant. All variables in the model are standardized, so $\beta$ represents standardized regression weights. $s r^{2}$ represents the semi-partial correlation squared. $L L$ and $U L$ indicate the lower and upper limits of a confidence interval, respectively. Statistically significant results are bolded for emphasis.

$*$ indicates $p<.05, * *$ indicates $p<.01$, *** indicates $p<.001$. 
Table S5

Regression Results Predicting Presence of and Search for Meaning, Excluding Atheists, Agnostics, and Participants with No Particular Religious Affiliation ( $\mathrm{N}=637)$

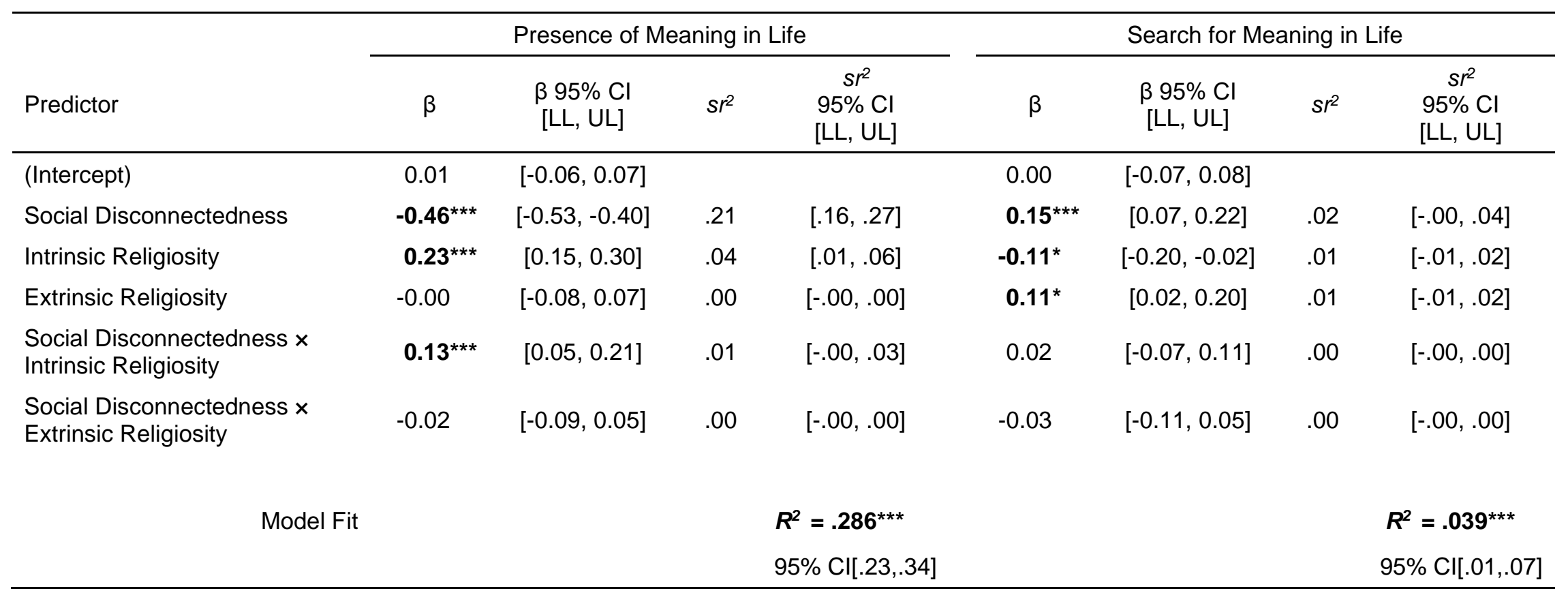

Note. A significant $\beta$-weight indicates the semi-partial correlation is also significant. All variables in the model are standardized, so $\beta$ represents standardized regression weights. $s r^{2}$ represents the semi-partial correlation squared. $L L$ and $U L$ indicate the lower and upper limits of a confidence interval, respectively. Statistically significant results are bolded for emphasis.

$*$ indicates $p<.05, * *$ indicates $p<.01, * * *$ indicates $p<.001$. 
Table S6

Regression Results Predicting Presence of and Search for Meaning Based on Alternative Scoring of Intrinsic and Extrinsic Religiosity

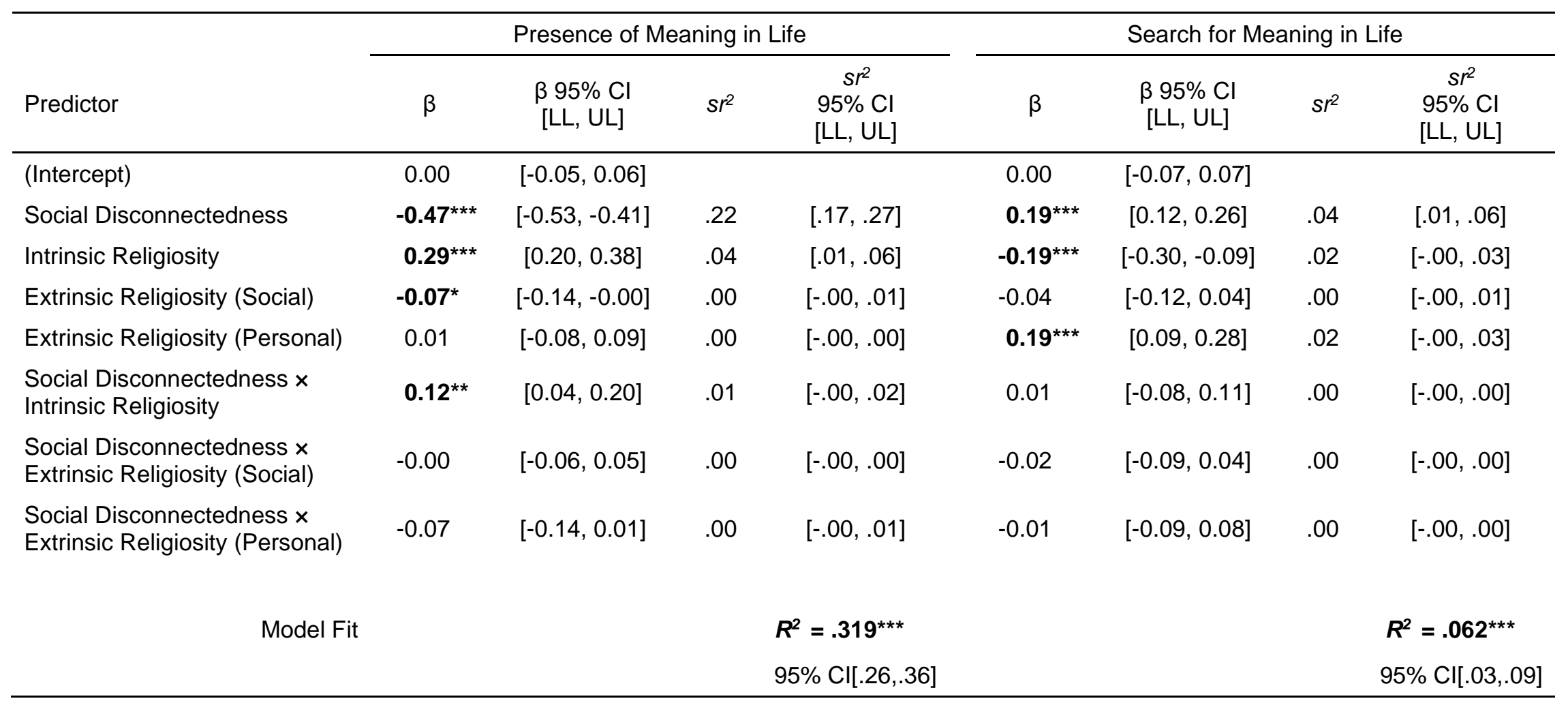

Note. A significant $\beta$-weight indicates the semi-partial correlation is also significant. All variables in the model are standardized, so $\beta$ represents standardized regression weights. $s r^{2}$ represents the semi-partial correlation squared. $L L$ and $U L$ indicate the lower and upper limits of a confidence interval, respectively. Statistically significant results are bolded for emphasis.

$*$ indicates $p<.05, * *$ indicates $p<.01, * * *$ indicates $p<.001$. 
Table S7

Regression Results Predicting Presence of and Search for Meaning Based on Alternative Scoring of Intrinsic and Extrinsic Religiosity, Excluding Atheists and Agnostics ( $\mathrm{N}=735)$

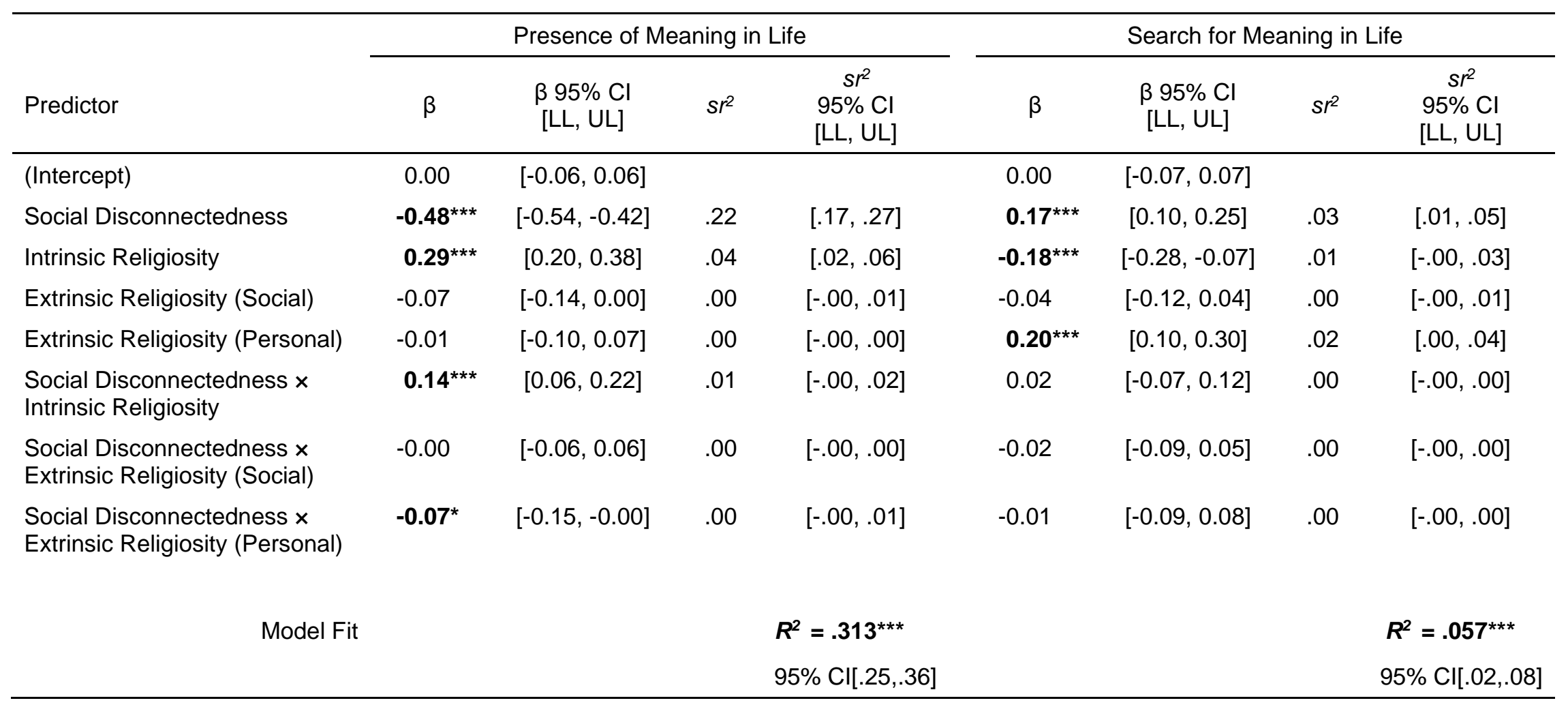

Note. A significant $\beta$-weight indicates the semi-partial correlation is also significant. All variables in the model are standardized, so $\beta$ represents standardized regression weights. $s r^{2}$ represents the semi-partial correlation squared. $L L$ and $U L$ indicate the lower and upper limits of a confidence interval, respectively. Statistically significant results are bolded for emphasis.

$*$ indicates $p<.05, * *$ indicates $p<.01$, *** indicates $p<.001$. 
Table S8

Regression Results Predicting Presence of and Search for Meaning Based on Alternative Scoring of Intrinsic and Extrinsic Religiosity, Excluding Atheists, Agnostics, and Participants with No Particular Religious Affiliation ( $\mathrm{N}=637)$

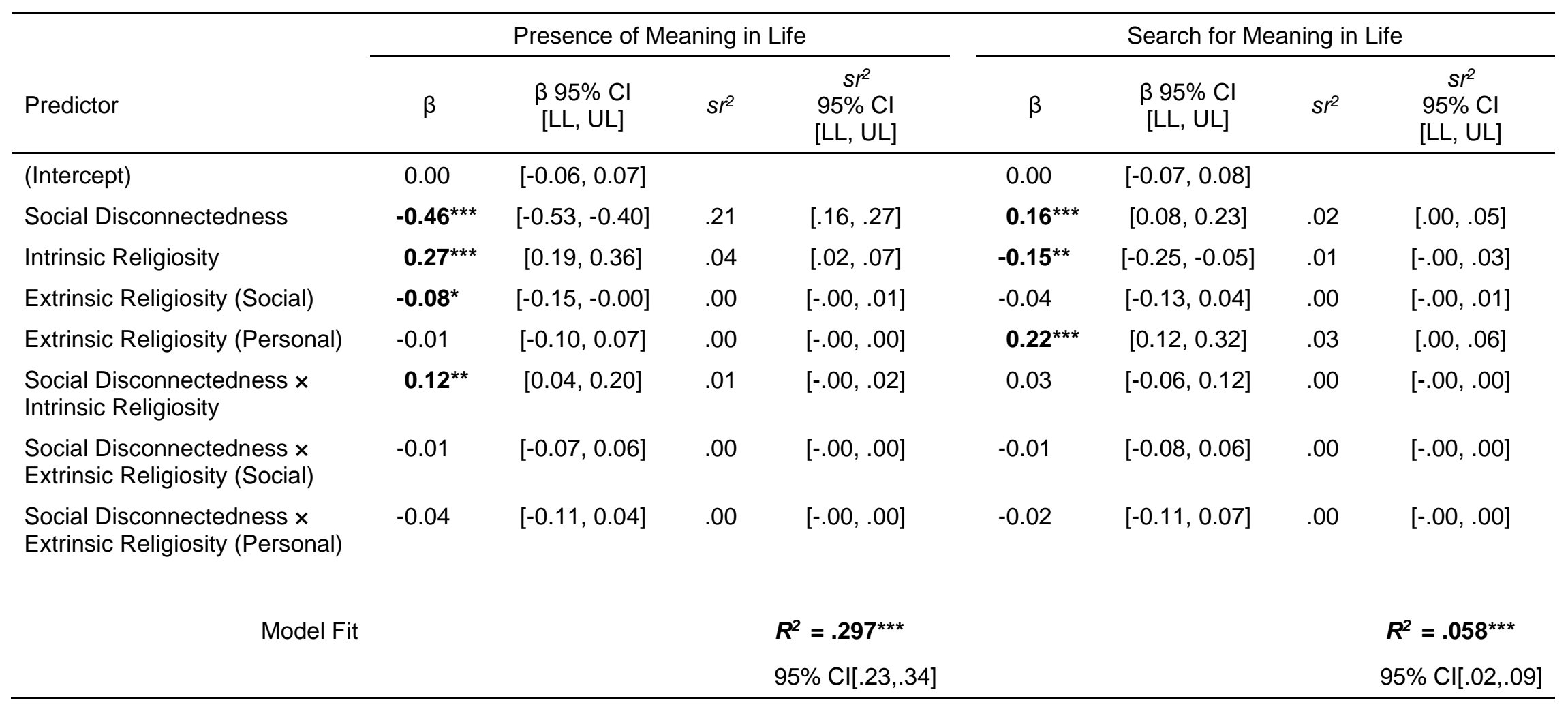

Note. A significant $\beta$-weight indicates the semi-partial correlation is also significant. All variables in the model are standardized, so $\beta$ represents standardized regression weights. $s r^{2}$ represents the semi-partial correlation squared. $L L$ and $U L$ indicate the lower and upper limits of a confidence interval, respectively. Statistically significant results are bolded for emphasis.

$*$ indicates $p<.05, * *$ indicates $p<.01, * * *$ indicates $p<.001$. 


\section{References}

Batson, C. D., \& Schoenrade, P. A. (1991). Measuring religion as quest: 1) Validity concerns. Journal for the Scientific Study of Religion, 30, 416-429.

Baumeister, R. F., \& Landau, M. J. (2018). Finding the meaning of meaning: Emerging insights on four grand questions. Review of General Psychology, 22, 1-10.

Baumeister, R. F., \& Leary, M. R. (1995). The need to belong: Desire for interpersonal attachments as a fundamental human motivation. Psychological Bulletin, 117, 497-529.

Chan, T., Michalak, N. M., \& Ybarra, O. (2019). When God is your only friend: Religious beliefs compensate for purpose in life in the socially disconnected. Journal of Personality, 87, 455-471.

Cohen, A. B., Mazza, G. L., Johnson, K. A., Enders, C. K., Warner, C. M., Pasek, M. H., \& Cook, J. E. (2017). Theorizing and measuring religiosity across cultures. Personality and Social Psychology Bulletin, 43, 1724-1736.

Darlington, R., \& Hayes, A. F. (2017). Regression analysis and linear models: Concepts, applications, and implementation. New York, NY: Guilford Press.

Faul, F., Erdfelder, E., Lang, A.-G., \& Buchner, A. (2007). G*Power 3: A flexible statistical power analysis program for the social, behavioral, and biomedical sciences. Behavior Research Methods, 39, 175-191.

Forsyth, D. P. (1980). A taxonomy of ethical ideologies. Journal of Personality and Social Psychology, 39, 175-184.

Fox, J., \& Weisberg, S. (2019). An R companion to applied regression (3rd ed.). Thousand Oaks, CA: Sage. Retrieved from https://socialsciences.mcmaster.ca/jfox/Books/Companion/ 
Goodwin, G. P., \& Darley, J. M. (2008). The psychology of meta-ethics: Exploring objectivism. Cognition, 106, 1339-1366.

Goodwin, G. P., \& Darley, J. M. (2010). The perceived objectivity of ethical beliefs:

Psychological findings and implications for public policy. Review of Philosophy and Psychology, 1, 161-188.

Goodwin, G. P., \& Darley, J. M. (2012). Why are some moral beliefs perceived to be more objective than others? Journal of Experimental Social Psychology, 48, 250-256.

Gorsuch, R. L., \& McPherson, S. E. (1989). Intrinsic/extrinsic measurement: I/E-Revised and single-item scales. Journal for the Scientific Study of Religion, 28, 348-354.

Gorsuch, R. L., \& Venable, G. D. (1983). Development of an “Age Universal” I-E Scale. Journal for the Scientific Study of Religion, 22, 181-187.

Hawkley, L. C., \& Cacioppo, J. T. (2010). Loneliness matters: A theoretical and empirical review of consequences and mechanisms. Annals of Behavioral Medicine, 40, 218-227.

Hayes, A. F. (2013). Introduction to mediation, moderation, and conditional process analysis: A regression-based approach (1st ed.). New York, NY: Guilford.

Hicks, J. A., \& King, L. A. (2009). Positive mood and social relatedness as information about meaning in life. The Journal of Positive Psychology, 4, 471-482.

Hicks, J. A., Schlegel, R. J., \& King, L. A. (2010). Social threats, happiness, and the dynamics of meaning in life judgments. Personality and Social Psychology Bulletin, 36, 1305-1317.

Lambert, N. M., Stillman, T. F., Hicks, J. A., Kamble, S., Baumeister, R. F., \& Fincham, F. D. (2013). To belong is to matter: Sense of belonging enhances meaning in life. Personality and Social Psychology Bulletin, 39, 1418-1427.

Lee, R. M., \& Robbins, S. B. (1995). Measuring belongingness: The Social Connectedness and 
the Social Assurance Scales. Journal of Counseling Psychology, 42, 232-241.

Long, J. A. (2018). Jtools: Analysis and presentation of social scientific data. Retrieved from https://cran.r-project.org/package=jtools

Maltby, J., \& Day, L. (1998). Amending a measure of the Quest religious orientation: Applicability of the scale's use among religious and non-religious persons. Personality and Individual Differences, 25, 517-522.

Maltby, J., \& Lewis, C. A. (1996). Measuring intrinsic and extrinsic orientation toward religion: Amendments for its use among religious and non-religious samples. Personality and Individual Differences, 21, 937-946.

Maltby, J., McCollam, P., \& Millar, D. (1994). Religiosity and obsessionality: A refinement. The Journal of Psychology, 128, 609-611.

Maitland, A. (2009). How many scale points should I include for attitudinal questions? Survey Practice, 2(5). Retrieved from https://www.surveypractice.org/article/2950

Pew Research Center. (2015). U.S. public becoming less religious: Modest drop in overall rates of belief and practice, but religiously affiliated Americans are as observant as before. Retrieved from http://assets.pewresearch.org/wpcontent/uploads/sites/11/2015/11/201.11.03_RLS_II_full_report.pdf

R Core Team. (2017). R: A language and environment for statistical computing. Vienna, Austria: R Foundation for Statistical Computing. Retrieved from https://www.R-project.org/

Reynolds, C. J., Gamez-Djokic, M., Molden, D., \& Conway, P. (in prep). Religiosity both increases and decreases deontological and utilitarian dilemma response inclinations: A process dissociation analysis and meta-analysis. 
Stanley, D. (2018). apaTables: Create American Psychological Association (APA) style tables. Retrieved from https://CRAN.R-project.org/package=apaTables

Steger, M. F., Frazier, P., Oishi, S., \& Kaler, M. (2006). The Meaning in Life Questionnaire: Assessing the presence of and search for meaning in life. Journal of Counseling Psychology, 53, 80-93.

Stillman, T. F., Baumeister, R. F., Lambert, N. M., Crescioni, A. W., DeWall, C. N., \& Fincham, F. D. (2009). Alone and without purpose: Life loses meaning following social exclusion. Journal of Experimental Social Psychology, 45, 686-694.

Wickham, H. (2017). Tidyverse: Easily install and load the 'tidyverse'. Retrieved from https://CRAN.R-project.org/package=tidyverse

Wickham, H., \& Miller, E. (2018). Haven: Import and export 'spss', 'stata' and 'sas' files. Retrieved from https://CRAN.R-project.org/package=haven 